\title{
Rigid spine syndrome
}

\author{
IKU O GOTO, S USUMU NAGASAKA, H ITOSHI NAG A R A \\ A N D Y OSH I G ORO K URO I W A \\ From the Department of Neurology, Neurological Institute, Faculty of Medicine, \\ Kyushu University, Fukuoka, Japan
}

SUMMARY A patient is described with distinctive clinical features including childhood onset of non-progressive muscle weakness, limitation of flexion of the neck and spine, scoliosis, and joint contracture. Muscle biopsy and electromyography showed findings suggestive of a myopathic disorder. The combination of these distinctive clinical features is termed the rigid spine syndrome. Only six cases have been reported in the literature to date.

The rigid spine syndrome which is characterised by limitation of neck and trunk flexion, scoliosis, mild degree of joint contractures, and mild diffuse nonprogressive weakness, has been described by several authors (Dubowitz, 1970, 1971; Dubowitz and Brooke, 1973; Goebel et al., 1977; Seay et al., 1977). In this paper we report an additional case.

\section{Case report}

A 16 year old Japanese boy was admitted to Kyushu University Hospital for evaluation of weakness of the extremities and limitation of movement of the spine. His mother had a normal pregnancy and delivery. He weighed $3100 \mathrm{~g}$ at birth, and he developed normally until age 3 years, when he began to run much more slowly than his friends. At age 6 years, limitation of neck and back flexion and weakness of extremities were noted. Progression of his symptoms was not noted. There was neither back nor neck pain. He was otherwise in good health throughout his childhood. His school performance was above average. His paternal grandparents were first cousins and were healthy. His two brothers and other relatives were all in good health.

Examination on admission showed a thin boy with a high arched palate and mild scoliosis. He had no intellectual impairment. His neck was hyperextended with limitation of flexion and lateral rotation. The spine was rigid but some degree of flexion and extension was possible (Fig. 1). There was no tenderness to spinal percussion and no

Address for reprint requests : Dr Ikuo Goto, Department of Neurology, Neurological Institute, Faculty of Medicine. Kyushu University 60. Fukuoka 812, Japan

Accepted 2 October 1978

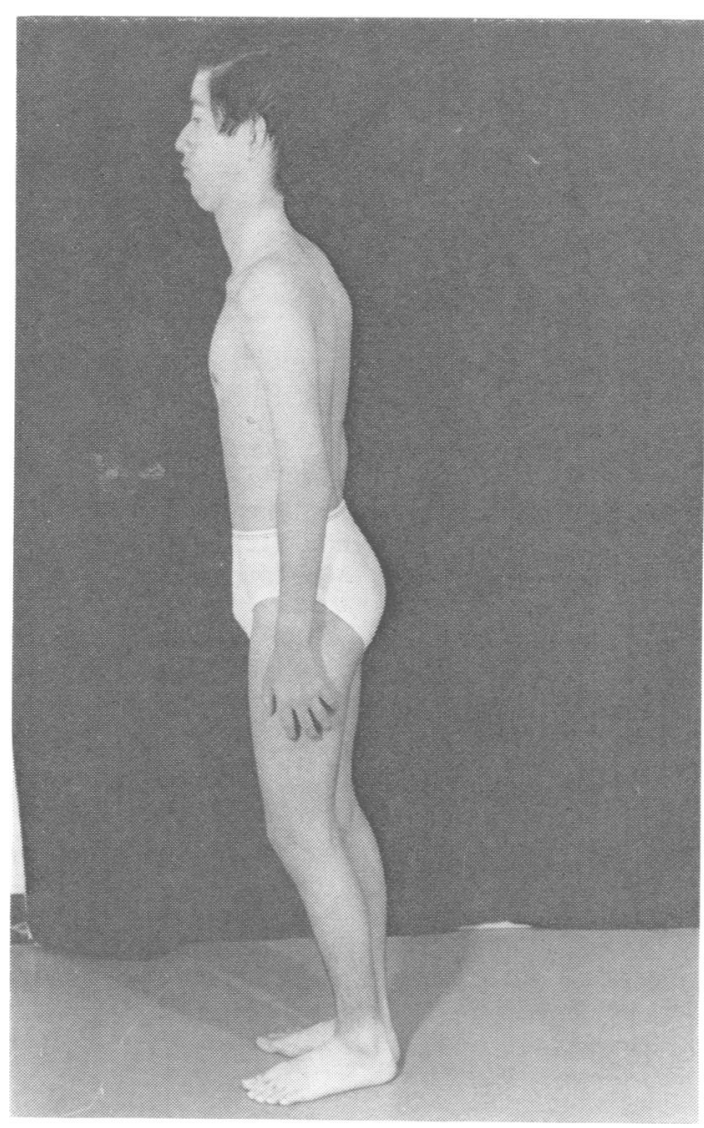

(a)

Fig. 1 Patient showing characteristic posture and contractures. Note limitation in flexion of the neck and spine in association with a good range of extension $(a, b, c)$. 


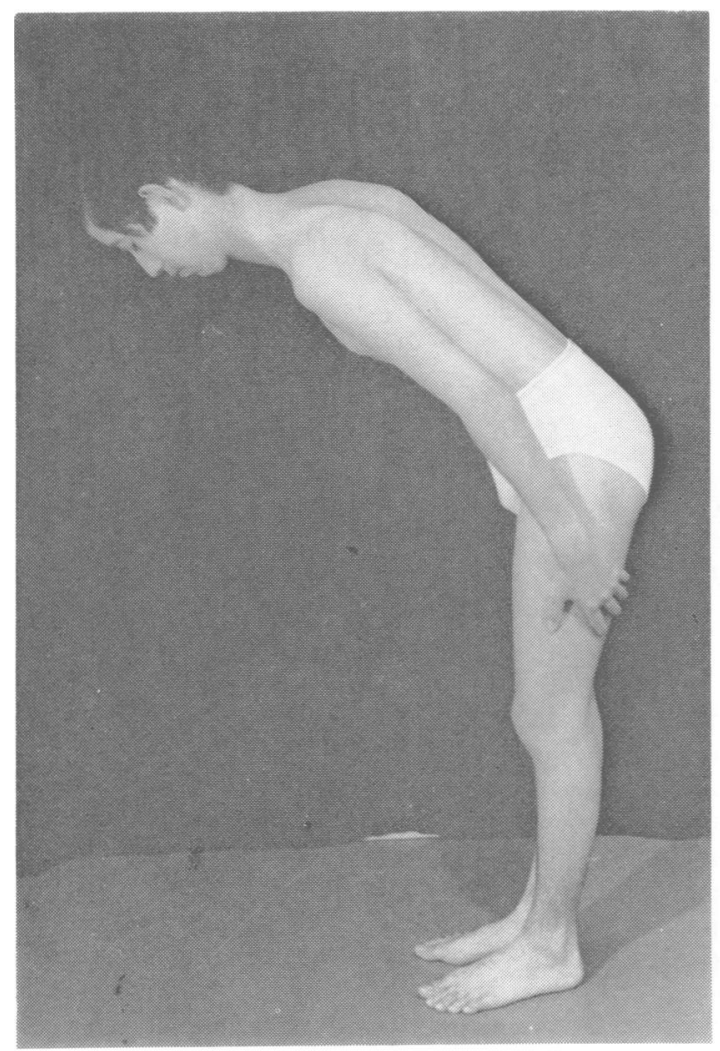

(b)

spontaneous pain. There was a mild degree of limitation of extension of both the elbows and the knees. The sternocleidomastoid muscles were extremely weak and atrophic. The cranial nerves were intact. There was a mild degree of muscular weakness and atrophy and hypoactive reflexes in all four extremities. Pathological reflexes were not elicited. Neither sensory impairment nor cerebellar signs were found.

Laboratory examination showed an increase of serum creatine phosphokinase level to $102 \mathrm{iu} / 1$ (normal level less than $20 \mathrm{iu} / \mathrm{l}$ ). The serological test for syphilis was negative. The following laboratory studies were normal: total blood count, urine, faeces, ESR, serum protein electrophoresis, electrolytes, cholesterol, phospholipid, triglycerides, aspartate and alanine transaminase, lactate dehydrogenase, alkaline phosphatase, blood urea nitrogen, thyroid function, urinary 17-oxosteroid and 17hydroxysteroid, glucose tolerance test, and CSF The ECG showed right axis deviation. Radiological examination showed a mild degree of scoliosis and absence of the normal lumbar and cervical lordosis.

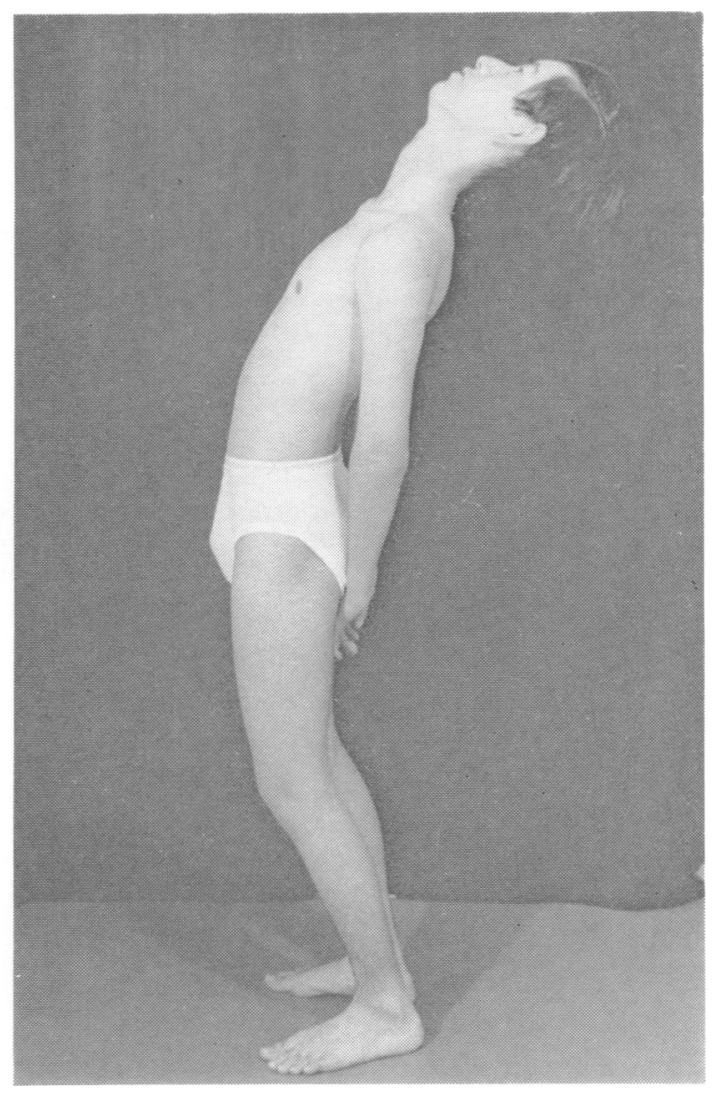

(c)

There was no ankylosis nor any destructive process of the spine. The deltoid, anterior tibial, and sternocleidomastoid muscles were examined by electromyography. There was no spontaneous discharge at rest. Motor unit action potentials were of low amplitude and short duration. There were full interference patterns. The motor conduction velocities of the right median and peroneal nerves were normal at $61.4 \mathrm{~m} / \mathrm{s}$ and $49.3 \mathrm{~m} / \mathrm{s}$, respectively. Muscle biopsy specimens of the right biceps showed mild to moderate variation in fibre size and mild increases in endomysial and perimysial connective tissues, and central nuclei were occasionally seen (Fig. 2). Reduced diphosphopyridine nucleotide and adenosine triphosphatase stains showed fibre size variability in both type 1 and type 2 fibres (Fig. 2). No mitochondrial abnormalities, ragged-red fibres, or inflammatory cells were seen. Electron microscopic examination showed no abnormality.

This patient has had no demonstrable worsening of his muscle strength or contractures after three years of follow-up. 

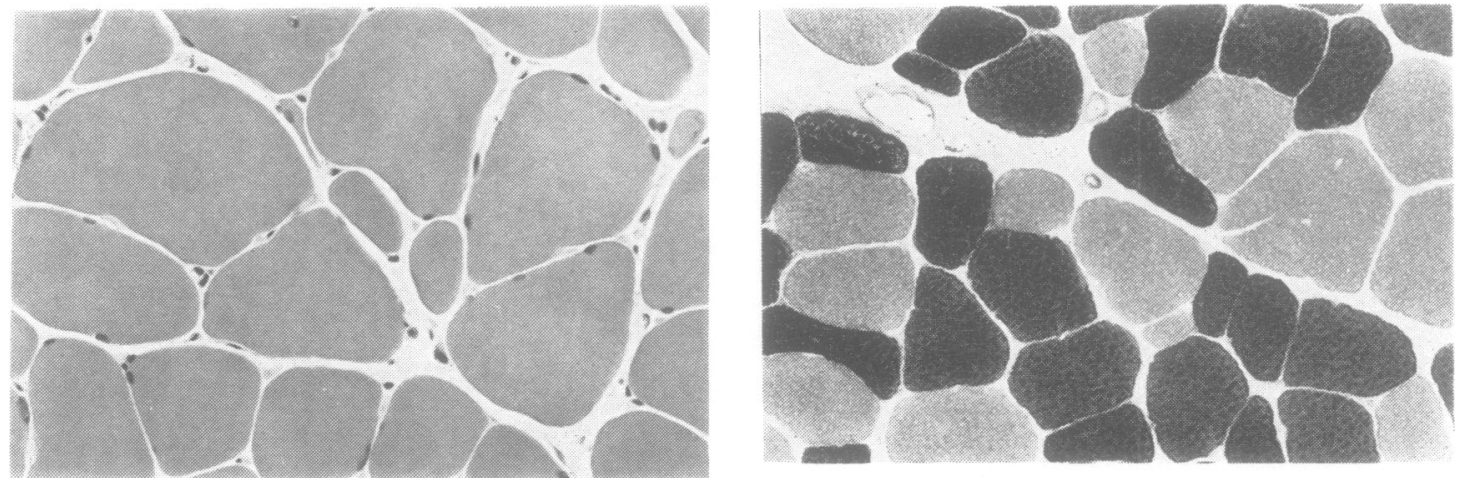

Fig. 2 Left: transverse section of the right biceps muscle showing variation in fibre size. Frozen section. Haematoxylin and eosin staining, original magnification $\times 270$. Right: ATPase stain at pH 9.4 shows a checkerboard distribution of both fibre types and variation in fibre size. Frozen section, original magnification $\times 210$.

\section{Discussion}

In early childhood our patient developed limitation of flexion and lateral rotation of trunk and neck, scoliosis, mild joint contractures, and mild non-progressive muscular weakness. The serum creatine phosphokinase level was moderately increased. The EMG and muscle biopsy suggested a myopathic disorder rather than a neurogenic one. Radiography showed no abnormalities of the bone or joint. The family history was negative for similar disorders. Several authors (Dubowitz, 1970, 1971; Dubowitz and Brooke, 1973; Goebel et al., 1977; Seay et al., 1977) have described a similar disorder, which is usually termed the rigid spine syndrome. Dubowitz $(1970,1971)$ and Dubowitz and Brooke (1973) reported four cases but clinical and laboratory findings were presented for only two. Seay et al. (1977) reported one case and Goebel et al. (1977) also reported a case. All cases on which we could get information were males, with onset from infancy to 7 years of age, limitation of flexion of the spine, joint contractures, mild diffuse muscular weakness, and scoliosis. None of them had any clear family history of a similar disease according to the reports. However, Rotthauwe et al. (1972) reported a family with a somewhat similar condition with sex-linked recessive inheritance which showed muscle weakness and wasting in a scapulo-humeral-distal distribution, limitation of flexion of the spine, flexion contractures in the elbow, hip, and foot, and cardiac arrhythmias. Serum creatine phosphokinase levels were normal or moderately raised, and the EMG showed a myopathic pattern in these cases. No bone and joint abnormalities were found except for curvature of the spine.
Although the reason for the limitation of flexion of the neck and trunk in these cases is not clear, fibrosis may be present in the axial muscles as suggested by Dubowitz and Brooke (1973) and Goebel et al. (1977). Dubowitz (1970, 1971) and Dubowitz and Brooke (1973) reported marked proliferation of endomysial and perimysial connective tissue, size variation of muscle fibres, internal nuclei, necrosis and phagocytosis in the various muscle specimens. On the other hand, Seay et al. (1977) reported a distinctive abnormality of the small type 1 fibres in the biceps muscle biopsy specimens. Goebel et al. (1977) also described marked endomysial fibrosis, occasional necrosis and phagocytosis, and internal nuclei in the erector trunci muscles, and type 1 fibre atrophy and predominance, hypertrophy of type 2 fibres, internal nuclei and endomysial fibrosis in the biceps muscle. In our patient, biceps muscle biopsy specimens showed fibre size variation and mild proliferation of endomysial and perimysial connective tissues without any abnormality of fibre type or mitochondria. Although the causes of the differences in muscle histology among these cases are obscure, this might be the result of a difference in the site of muscle biopsies or the stage of disease.

In spite of muscle biopsy differences, our case and the six previously described have a strikingly similar clinical picture. Therefore, this syndrome seems to be a distinct clinical entity characterised by non-progressive muscle weakness, limitation of flexion of the neck and spine, scoliosis, and joint contracture.

\section{References}

Dubowitz, V. (1970). Some unusual neuromuscular 
disorders. In Muscle Diseases. Proceedings of an International Congress (Milan, 19-21 May 1969), pp. 568-573. Edited by J. N. Walton, N. Canal, and G. Scarlato. Excerpta Medica: Amsterdam.

Dubowitz, V. (1971). Recent advances in neuromuscular disorders. Rheumatology and Physical Medicine, 11, 126-130.

Dubowitz, V., and Brooke, M. H. (1973). Rigid spine syndrome. In Muscle Biopsy. A Modern Approach, pp. 368-371. W. B. Saunders: London.

Goebel, H. H., Lenard, H. G., Görke, W., and Kunze,
K. (1977). Fibre type disproportion in the rigid spine syndrome. Neuropädiatrie, 8, 467-477.

Rotthauwe, H. W., Mortier, W., and Beyer, H. (1972). Neuer Typ einer recessiv X-chromosomal vererbten Muskeldystrophie: Scapulo-humero-distale Muskeldystrophie mit frühzeitigen Kontrakturen und Herzrhythmusstörungen. Humangenetik, 16, 181200.

Seay, A. R., Ziter, F. A., and Petajan, J. H. (1977). Rigid spine syndrome. A type 1 fiber myopathy. Archives of Neurology (Chicago), 34, 119-122. 